\title{
Nefritis tubulointersticial aguda. Revisión bibliográfica
}

\author{
John Fredy Nieto-Ríos' ${ }^{1}$ Luis Miguel Ruiz-Velásquez², Laura Álvarez³ , Lina María Serna-Higuita
}

\section{RESUMEN}

La nefritis tubulointersticial aguda hace referencia a un tipo de daño renal que afecta principalmente el intersticio y ocasiona la lesión renal aguda, potencialmente reversible. Su curso puede ser subclínico, con deterioro progresivo hasta evolucionar hacia la insuficiencia renal crónica. La nefritis tubulointersticial aguda tiene múltiples etiologías, las más frecuentes son los medicamentos, productos herbales, las infecciones y las enfermedades autoinmunes. Las principales manifestaciones clínicas son la poliuria, polaquiuria, nocturia, dolor lumbar, microhematuria y leucocituria, aunque puede ser totalmente asintomática. El tratamiento depende de la causa de base y los esteroides juegan un papel importante cuando la condición es de oriogen medicamentoso o autoinmune. El pronóstico generalmente es bueno, si el problema se identifica de forma oportuna y se trata adecuadamente.

\section{PALABRAS CLAVE}

Enfermedades Renales; Insuficiencia Renal; Lesión RenaI Aouda; Nefritis TubulointersticiaI

\section{SUMMARY}

\section{Acute interstitial nephritis. Literature review}

Acute tubulointerstitial nephritis is a kidney lesion that mainly affects the interstitium and can lead to a reversible acute kidney injury. It can progress subclinically, with progressive development towards chronic renal failure. Acute tubulointerstitial nephritis has several causes, being the most common medications, herbal products, infections and autoimmune diseases. The main clinical manifestations are polyuria, polaquiuria, nycturia, lumbar pain,

\footnotetext{
Médico Internista, Nefrólogo Hospital Pablo Tobón Uribe - Universidad de Antioquia, Medellín, Colombia.

Médico Residente de Medicina Interna, Universidad Pontificia Bolivariana, Medellín, Colombia.

Médico General, Fundación Universitaria San Martín, Medellín, Colombia.

Estudiante de doctorado, Departamento de Epidemiología clínica y Biometría. Universidad de Tübingen, Alemania.
}

Correspondencia: John Fredy Nieto-Ríos; johnfredynieto@gmail.com

Recibido: agosto 14 de 2019

Aceptado: 21 de octubre de 2019

Cómo citar: Nieto-Ríos JF, Ruiz-Velásquez LM, Álvarez L, Serna-Higuita LM. Nefritis tubulointersticial aguda. Revisión bibliográfica. latreia. 2020Abr-Jun;33(2):155-166. D0I 10.17533/udea.iatreia.45. 
microhematuria, leukocyturia, although the patients also can be completely asymptomatic. Treatment is determined by the underlying cause. Steroids play an important role when tubulointerstitial nephritis is caused by medication or autoimmune diseases. The prognosis is usually good if the problem is detected early and treated properly.

\section{KEY WORDS}

Acute Renal Injury; Kidney Diseases; Nephritis, Interstitial; Renal Insufficiency

\section{INTRODUCCIÓN}

La nefritis tubulointersticial aguda (NTIA) es un tipo de lesión renal que afecta típicamente el intersticio y se acompaña, generalmente, de daño tubular ${ }^{(1)}$. Se caracteriza por un infiltrado inflamatorio en el intersticio del riñón asociado a un deterioro de la función renal ${ }^{(2,3)}$.

Aunque la incidencia de la NTIA no es tan alta como otros tipos de lesión renal aguda (LRA), su conocimiento es relevante, ya que el diagnóstico temprano de esta enfermedad, aunado a un tratamiento adecuado, puede evitar la pérdida definitiva de la función renal ${ }^{(4)}$.

Entre los mecanismos fisiopatológicos que explican la NTIA se encuentran las reacciones de hipersensibilidad intersticial con afección tubular renal secundarias al uso de fármacos, las infecciones y las enfermedades autoinmunes ${ }^{(5)}$. Esta revisión pretende evaluar el estado del arte de la NTIA, para así permitir al clínico adquirir un conocimiento actualizado sobre la fisiopatología, la evolución clínica, el diagnóstico y el tratamiento de esta enfermedad.

\section{EPIDEMIOLOGÍA}

La NTIA es el diagnóstico histopatológico presente entre el 1 y el $3 \%$ de las biopsias renales ${ }^{(6-9)}$, pero en pacientes con LRA que son llevados a biopsia renal, este porcentaje se incrementa del 13 al $27 \%{ }^{(6,10,11)}$.

En la práctica clínica, la verdadera incidencia de la NTIA está subestimada, esto debido a la pobre sospecha diagnóstica de los clínicos en escenarios de la LRA y a la poca disponibilidad de la biopsia renal en algunos centros ${ }^{(6)}$.

La literatura reciente sugiere que la incidencia de la NTIA podría estar en aumento por el uso indiscriminado de fármacos (principalmente AINES y antibióticos), la polifarmacia y el consumo de productos herbales; con una mayor susceptibilidad en los ancianos $^{(12,13)}$.

EI registro español de biopsias renales durante el período 1994-2009, estimó una prevalencia de la NTIA del 3,6 \% en los primeros 4 años, encontrando una prevalencia de más del doble (10,5\%) en los siguientes años estudiados. Dicho incremento fue mayor en los pacientes ancianos (> 65 años), oscilando desde un 1,6 hasta un $12,3 \%$, hallazgos reportados previamente en otros estudios ${ }^{(10,12,14-16)}$.

Adicionalmente, en la población anciana se observa una mayor frecuencia de la NTIA inducida por medicamentos en comparación con los jóvenes, sus desenlaces son peores en cuanto al aumento de azoados, la necesidad de terapia dialítica y las secuelas como la enfermedad renal crónica (ERC) ${ }^{(13)}$.

\section{ETIOLOGÍA}

La etiología de la NTIA ha cambiado con el paso del tiempo y con la introducción de nuevos medicamentos. En la actualidad, se conoce que cerca del 70 \% de las NTIA son secundarias al uso de fármacos ${ }^{(17)}$. Sin embargo, un gran porcentaje de estas es causado por infecciones, tóxicos, productos herbales y enfermedades autoinmunes ${ }^{(12,18,19)}$.

Aunque existe poco conocimiento de los productos herbales como causa de NTIA, se sabe que el ácido aristolóquico, presente en muchas hierbas chinas, ocasiona este tipo de afección. Adicionalmente, existen muchos otros productos utilizados por la población en general de los cuales se desconoce su potencial de producir Ia NTIA.

La clasificación de la Organización Mundial de Ia SaIud para las enfermedades tubulointersticiales tiene en cuenta la etiología, clínica y las características histológicas (Tabla 1) ${ }^{(20,21)}$. 
Tabla 1. Clasificación de las enfermedades tubulointersticiales

\begin{tabular}{|c|}
\hline $\begin{array}{l}\text { Clasificación de las enfermedades tubulointersticiales } \\
\text { (OMS) }\end{array}$ \\
\hline Infecciones: \\
\hline Nefritis tubulointersticial infecciosa aguda \\
\hline NTI aguda asociada a la infección sistémica \\
\hline NTI infecciosa crónica (pielonefritis crónica) \\
\hline Infecciones renales específicas \\
\hline NTI inducidas por medicamentos: \\
\hline Lesión tubular tóxica aguda \\
\hline NTI por hipersensibilidad \\
\hline NTI crónica \\
\hline $\mathrm{NTI}$ asociada con desórdenes inmunes \\
\hline $\begin{array}{l}\text { Inducida por anticuerpos que reaccionan con antígenos } \\
\text { tubulares }\end{array}$ \\
\hline Inducida por complejos inmunes autólogos o exógenos \\
\hline $\begin{array}{l}\text { Inducida por, o asociada con, la hipersensibilidad me- } \\
\text { diada por células }\end{array}$ \\
\hline Inducida por la hipersensibilidad inmediata (IgE) \\
\hline
\end{tabular}

Fuente: adaptación Arias LF., y Fogo AB et al

\section{Medicamentos}

Cualquier medicamento, teóricamente, podría causar NTIA. En la práctica clínica se han reportado más de 150 compuestos diferentes que conllevan al desarrollo de esta entidad ${ }^{(22)}$. Incluso la combinación de varios productos podría aumentar la susceptibilidad para desarrollarla. Entre los medicamentos más implicados con la NTIA están los AINES, Ios inhibidores de la bomba de protones, los betalactámicos, la ciprofloxacina, los antituberculosos (rifampicina, isoniazida, pirazinamida), los anticonvulsivantes (fenitoína), las sulfas, el captopril, la furosemida, el alopurinol y Ios aminosalicilatos, entre otros.

AINES. La severidad del compromiso de la NTIA por AINES es variable ${ }^{(15)}$. Frecuentemente, su desarrollo es lento con manifestaciones que pueden aparecer varias semanas posteriores a la ingesta del fármaco. Histológicamente, se caracteriza por un infiltrado eosinofílico del intersticio renal ${ }^{(23)}$. Entre los hallazgos de laboratorio se encuentra desde la eosinofiluria hasta la proteinuria en rango nefrótico, por su asociación adicional con la enfermedad de cambios mínimos. En la mayoría de casos, el compromiso renal revierte posterior a la suspensión del fármaco. Sin embargo, en algunos casos pueden quedar secuelas como el deterioro permanente de la función renal.

Cualquier tipo de AINES puede precipitar el daño intersticial renal, no obstante, existe una fuerte relación del fenoprofeno con la presencia de la NTIA. Adicionalmente, la combinación de AINES con otros analgésicos puede incrementar la posibilidad de desarrollar NTIA ${ }^{(2)}$.

Antibióticos $\beta$-lactámicos. Estos medicamentos son el principal causante de la NTIA reportado en Ia Iiteratura desde el año $1940{ }^{(24)}$. Específicamente, la meticilina se asocia con el síndrome de hipersensibilidad renal, por lo cual su uso en la actualidad no es recomendado. Sin embaroo, el compromiso del túbulo-intersticio renal ha sido reportado con otros $\beta$-lactámicos, incluyendo cefalosporinas. La duración de la exposición para el desarrollo de la NTIA es usualmente corta (pocos días o semanas). Del 30 al $40 \%$ de los pacientes expuestos a $\beta$-lactámicos que desarrollan la NTIA presentan la triada clásica: erupción cutánea (rash), eosinofilia y fiebre, sumada a los hallazosos patológicos típicos en el uroanálisis (hematuria, leucocituria y proteinuria) ${ }^{(24)}$. Su curso usualmente es limitado sin progresión a la ERC, siempre que se diagnostique oportunamente, se suspenda el fármaco implicado y se trate adecuadamente ${ }^{(25)}$; de lo contrario, puede quedar como secuela la ERC de severidad variable.

Antimicrobianos no $\beta$-lactámicos. Diversas sustancias antimicrobianas se han asociado a la NTIA. Entre el grupo de antibióticos no $\beta$-lactámicos, la ciprofloxacina presenta un gran número de reportes de caso; esto no sucede con otras fluoroquinolonas, por lo cual parece no existir efecto de clase.

Las sulfonamidas pueden causar NTIA en cualquier persona, pero afectan principalmente a pacientes inmunocomprometidos por trasplante o por VIH ${ }^{(24)}$.

La vancomicina asociada con la necrosis tubular aguda puede inducir a la hipersensibilidad e incluso, desarrollar granulomas no infecciosos que también están descritos en el consumo de penicilinas, nitrofurantoína y doxiciclina.

Los antiretrovirales como el tenofovir, indinavir o atazanavir, además de causar daño renal directo u obstrucción tubular, pueden desencadenar reacciones de 
hipersensibilidad túbulo-intersticial sin asociarse con síntomas sistémicos ${ }^{(24,26)}$.

Inhibidores de bomba de protones. Son medicamentos de uso frecuente en la población general. De ellos existen reportes frecuentes de la NTIA desde que se inició su utilización en los años 90. La incidencia de la NTIA por inhibidores de bomba de protones (IBP) es alrededor de 2-20 por cada 100.000 habitantes ${ }^{(24)}$. EI intervalo de tiempo entre el consumo de estos medicamentos y la presencia de la NTIA es bastante laroo, oscilando entre un mínimo de 15 días hasta un máximo de 18 meses, con un promedio de 10 a 12 semanas después del consumo de ellos.

Por su uso extendido, algunos estudios posicionan a Ios IBP como la segunda causa de la NTIA por fármacos, siendo los responsables del 14 al $64 \%$ de los casos de ella ${ }^{(25,27,28)}$. A diferencia de lo que sucede con los $\beta$-lactámicos, el compromiso intersticial renal por los IBP rara vez se presenta asociado a los clásicos síntomas de hipersensibilidad (menos del $10 \%$ tienen rash, fiebre o eosinofilia); el pronóstico es relativamente bueno si se descubre tempranamente y se suspende este tipo de medicamentos ${ }^{(12,24)}$.

Aminosalicilatos. Utilizados en el manejo de la enfermedad inflamatoria intestinal, estos medicamentos han sido relacionados con patologías renales entre las que se resaltan la NTIA y la necrosis tubular aguda. También se ha encontrado nefropatía por IogA, pero esta, es más asociada con la enfermedad de base que a este tipo de medicamentos ${ }^{(29)}$. La incidencia de la NTIA relacionado con el consumo de aminosalicilatos es de 1 por cada 200 a 500 individuos expuestos. Sin embarogo, existe el riesgo también del compromiso renal como manifestación extraintestinal de la enfermedad inflamatoria intestinal ${ }^{(30.31)}$. En estos pacientes se debe vigilar atentamente la presencia del rash y la eosinofilia que indican el compromiso intersticial renal, ya que la suspensión del medicamento es necesaria ${ }^{(31)}$.

\section{NTIA por infecciones}

Las infecciones fueron una de las causas más frecuentes de la NTIA en el pasado. Sin embargo, en la actualidad su incidencia ha disminuido de manera considerable ${ }^{(12)}$. Los mecanismos fisiopatológicos que explican el desarrollo de la NTIA secundaria a infecciones son variados, incluyendo el compromiso directo del parénquima renal (pielonefritis) o los factores inmunes derivados de la exposición a los antígenos microbianos ${ }^{(4)}$. Entre los microorganismos relacionados con la presencia de la NTIA se destacan: la Legionella, Leptospira, cytomegalovirus (CMV), Streptococcus, Mycobacterium tuberculosis, Corynebacterium diphtheriae, virus de Epstein-Barr (EBV), Yersinia, Polyomavirus, Enterococcus, Escherichia coli, adenovirus, virus BK y Candida.

Los pacientes infectados por VIH son particularmente susceptibles al desarrollo de las enfermedades de hipersensibilidad renal, no solo por el compromiso infeccioso derivado directamente por el genoma viral, sino por las coinfecciones y el uso constante de diferentes medicamentos (antiretrovirales, antimicrobianos y medicamentos antituberculosos) $)^{(3,12,32,33)}$. En un estudio en donde se revisaron 262 biopsias renales de pacientes con $\mathrm{VIH}$, se encontró que cerca del $11 \%$ de ellos presentaron la NTIA sin compromiso de la función renal; de estos, en el 72 \% la causa de la NTIA fue asociada con medicamentos, especialmente, AINES y trimetoprim sulfametoxazol ${ }^{(32) \text {. }}$

Los pacientes inmunosuprimidos, principalmente los trasplantados, pueden tener una NTIA asociada con nefropatía por virus BK que debe ser documentada oportunamente para evitar el daño renal permanente ${ }^{(20,34)}$.

\section{NTIA asociada con enfermedades autoinmunes}

Se asocia principalmente a LES, sarcoidosis y síndrome de Sjögren. La principal manifestación renal del compromiso por Sjögren es la NTI crónica. Sin embargo, también se describe acidosis tubular renal y necrosis tubular aguda ${ }^{(35)}$. La NTIA es una manifestación importante de la sarcoidosis, el reconocimiento de granulomas no caseificantes asociado con las características inflamatorias del compromiso renal por sarcoidosis configuran el diagnóstico; estos pacientes usualmente responden muy bien al manejo inmunosupresor ${ }^{(12)}$.

Otras enfermedades como las vasculitis (típicamente se describe la poliarteritis nodosa) y la enfermedad por Igg4, pueden presentarse asociada a la NTIA. Esta última acompañada de hipocomplementemia, pancreatitis y en algunos casos glomerulonefritis membranosa.

\section{Síndrome de nefritis intersticial y uveítis (TINU)}

Descrito por primera vez en el año 1975 por Dobrin et aI., se considera una causa poco frecuente de NTIA. 
Hasta la actualidad se han reportado aproximadamente 200 casos en la literatura. Es más frecuente en mujeres, con una relación entre mujer y varón de 3:1, la edad promedio de presentación es de 15 años y se caracteriza por uveítis, síntomas sistémicos y la NTIA ${ }^{(36)}$.

\section{FISIOPATOLOGÍA DE LA NTIA}

La NTIA se considera una reacción de hipersensibilidad tardía idiosincrática a los antígenos endógenos nefritogénicos o a los exógenos procesados por las células tubulares ${ }^{(2,22)}$. Diferentes medicamentos pueden unirse a un componente normal de la membrana basal tubular comportándose como un hapteno o simular una molécula antigénica normalmente presente en la membrana basal tubular, induciendo una respuesta inmune contra este antígeno. Se piensa que algunos péptidos microbianos pueden ocasionar la NTIA a través de este mecanismo. El hecho de que solo pocos pacientes presenten una nefritis intersticial al sufrir una enfermedad infecciosa hace pensar que la expresión de antígenos nefritogénicos en el riñón es neutralizada por mecanismos complejos que probablemente involucren las células T supresoras ${ }^{(12)}$.

La mayoría de los medicamentos se caracterizan por ser moléculas pequeñas (menos de mil daltons), por lo cual no presentan propiedades inmunogénicas. Sin embargo, estas moléculas pueden unirse a proteínas transportadoras y volverse inmunogénicas en un proceso llamado haptenización. Los complejos hapteno-transportador tienen la capacidad de activar una respuesta inmune, la cual puede presentarse en la circulación que llega directamente al riñón ${ }^{(4,12)}$. En algunos casos, el medicamento actúa como un prohapteno y requiere ser metabolizado a un compuesto reactivo que se fija a las proteínas determinadas para realizar la haptenización, iniciánđose así una reacción de hipersensibilidad inmunológica ${ }^{(37)}$.

La respuesta inmunológica en la NTIA ha sido descrita en diferentes fases que se superponen. La primera fase consta del reconocimiento y presentación del antígeno, en la cual las células intersticiales peritubulares y las células epiteliales lesionadas funcionan como células presentadoras de antígenos. Este reconocimiento de antígenos induce una respuesta necroinflamatoria que se caracteriza por la liberación de citoquinas, incremento de la permeabilidad vascular y reclutamiento de células inflamatorias, lo que lleva a la muerte de las células tubulares renales (necroptosis) ${ }^{(9.22)}$. Esta fase inicial es seguida por una integrativa o reguladora de la respuesta inmune, donde tienen lugar eventos protectores que regulan la respuesta inflamatoria y la autolimitan. En esta, las células T supresoras y la subregulación de la expresión de moléculas clase II de histocompatibilidad (MHC class II) juegan un papel importante para el control de la respuesta inmune ${ }^{(24)}$.

Si persiste el estímulo o este fue muy intenso, las células epiteliales tubulares $y$ endoteliales inducen la producción de citoquinas que aumentan la expresión de fibroblastos, macrófagos y neutrófilos, los cuales, junto con la liberación de elastasas, colagenasas y especies reactivas de oxígeno, perpetúan el daño ocasionado por los linfocitos T, generando la lesión tubular (tubulitis) y la proliferación de matriz celular en lugar de remoción, lo que magnifica el proceso fibrótico ${ }^{(22,24)}$.

En la tercera fase, o fase efectora, la interacción de las células infiltrantes y las células renales pueden llevar a suprimir la respuesta inflamatoria en las formas leves o la pueden amplificar en las graves. La duración y gravedad de esta última fase depende de si la fuente antigénica es erradicada (infección) o descontinuada, en el caso de medicamentos ${ }^{(22)}$.

\section{MANIFESTACIONES CLÍNICAS DE LA NTIA}

Por ser la NTIA un síndrome que involucra la inmunidad celular, clínicamente se asocia con manifestaciones sistémicas de hipersensibilidad como la erupción cutánea, fiebre y eosinofilia ${ }^{(1)}$, tríada que está presente en el $20 \%$ de los pacientes con la NTIA secundaria a $\beta$-lactámicos y en menos del $10 \%$ de aquellos con la NTIA secundaria a otras etiologías ${ }^{(4,5,38)}$.

Usualmente, los hallazogos clínicos de la NTIA son variados e inespecíficos y lo más característico es la elevación de azoados. Se puede presentar astenia, adinamia, náuseas, vómito, además de las alteraciones en el volumen urinario (usualmente poliuria y, en los casos más graves, oliguria o anuria). La polaquiuria y la nocturia son frecuentes, debido a la alteración en la concentración de la orina (hipostenuria). También puede darse un síndrome de Fanconi que se caracteriza por 
acidosis tubular tipo 2, glucosuria, fosfaturia y aminoaciduria ${ }^{(20,39)}$. Otros síntomas reportados hasta en el $30 \%$ de los pacientes son la presencia de dolor en el flanco renal, reflejo del edema y la distensión de la cápsula renal ${ }^{(22,40)}$. Hasta en el $15 \%$ se puede presentar hematuria macroscópica, los síntomas articulares (artralgias) están presentes aproximadamente en el $25 \%$ de los $\operatorname{casos}^{(40)}$.

\section{ESTUDIOS DIAGNÓSTICOS EN LA NTIA}

En todo paciente con sospecha de la NTIA se debe realizar una serie de estudios básicos que incluyen BUN, creatinina, uroanálisis, relación proteinuria/ creatinuria, eosinófilos en orina, hemoleucograma, velocidad de sedimentación globular (VSG), proteína $C$ reactiva (PCR) y ecografía renal. Con lo anterior, se enfoca al paciente y se determina la necesidad de una biopsia renal y el tratamiento específico.

Las principales manifestaciones de laboratorio y de imagen en la NTIA son:

- Elevación de azoados: elevación temprana, incluso en pacientes sin poliuria, derivada del daño tubular e intersticial ${ }^{(22)}$.

- Eosinofilia: presente del 10 al 30 \% de los pacientes con la NTIA. Se debe descartar causas parasitarias, alérgicas y neoplásicas como el diagnóstico diferencial.

- Sedimento urinario: se puede observar leucocituria, microhematuria e incluso, cilindros leucocitarios; estos últimos son muy característicos de la NTIA pero también se pueden presentar en otras patologías como en la necrosis tubular aguda o en el síndrome nefrítico. ${ }^{(24,41)}$.

- Eosinófilos en orina: con sensibilidad cercana al 60 \% y una especificidad del $85 \%$, esta manifestación es relevante cuando se encuentran en más de $1 \%$ de las células, y verdaderamente importante con un valor mayor al $5 \%$ de eosinófilos en la orina por tinción de Hansen. Sin embargo, no es específica de la NTIA, pudiéndose presentar en otras patologías como la enfermedad ateroembólica, cistitis intersticial, neoplasias uroteliales y las infecciones del tracto urinario ${ }^{(12,22)}$.

- Hipostenuria y poliuria: secundarios al daño corticomedular e intersticial que conlleva a la alteración de la capacidad de concentración de la orina y al desarrollo de diabetes insípida nefrogénica ${ }^{(22,42)}$.

- Bicarbonaturia, glucosuria y aminoaciduria: similar al síndrome de Fanconi, por compromiso tubular extenso que también pueden observarse en la necrosis tubular aguda ${ }^{(20)}$.

- Proteinuria: variable, usualmente no nefrótica. En la NTIA por AINES es usual encontrar niveles de proteinuria mayores a 3 gramos por la asociación con la enfermedad de cambios mínimos ${ }^{(22)}$.

- Otros marcadores (N-GAL, KIM-1, cistatina C, TIMP2): en la actualidad se está empezando a implementar el uso de moléculas, recientemente descubiertas, que expresan el daño renal temprano. Sin embargo, ninguna de estas moléculas es específica para el diagnóstico de la NTIA y solo describen la presencia de la lesión renal aguda con compromiso tubular ${ }^{(43)}$.

- Ecografía: en ausencia de la enfermedad renal crónica sobreagregada, en la NTIA el tamaño renal está conservado o incluso aumentado, por un edema considerable del tejido ${ }^{(19,22)}$.

- Gammagrafía con Galio 67: un incremento de captación de Galio 67 se ha encontrado en pacientes con la NTIA, hallazgo no específico, ya que se observa en pacientes con pielonefritis, cáncer y glomerulopatías ${ }^{(4)}$.

Otros estudios complementarios se deben ejecutar cuando se sospechan enfermedades específicas (Tabla 2).

\section{Biopsia renaI}

La biopsia renal es el estándar de oro para el diagnóstico de la NTIA, se sugiere la realización de la biopsia renal en los siguientes casos:

- Pacientes con deterioro de la función renal, con uroanálisis compatible con la NTIA, pero sin encontrar medicamento sospechoso.

- Deterioro de la función renal, con uso de medicamento compatible con la NTIA y uroanálisis no patológico.

- Pacientes con la NTIA en los cuales se piensa iniciar manejo con glucocorticoides.

- Enfermos con la NTIA con baja respuesta clínica aI tratamiento. 
Tabla 2. Estudios complementarios sugeridos en el abordaje diagnóstico de los pacientes con nefritis tubulointersticial aguda

\begin{tabular}{ll}
\hline \multicolumn{1}{c}{ Estudios sugeridos } & \multicolumn{1}{c}{ Sospecha diagnóstica } \\
Rayos X de tórax & Tuberculosis u otras infecciones \\
\hline Niveles séricos de ECA, calcio sérico y urinario & Sarcoidosis \\
\hline PPD & Tuberculosis \\
\hline ANCA & Sospecha de la NTIA por autoinmunidad, vasculitis de pequeños vasos \\
\hline Anti-DNA, ENAS & Sospecha de lupus eritematoso o Sjögren \\
\hline Factor reumatoide y Anti-CCP & Sospecha de NTIA secundaria a artritis reumatoide \\
C3 y C4: & Cuando están bajos, se debe sospechar NTIA por autoinmunidad \\
\hline Electroforesis de proteínas & Sospecha de la NTIA por gammapatías monoclonales \\
\hline
\end{tabular}

ECA: enzima convertidora de angiotensina. PPD: prueba cutánea de tuberculina. CMV: citomegalovirus. VEB: virus Epstein Barr. ANCA: anticuerpos anticitoplasmáticos del neutrófilo. ENAS: antígenos nucleares extraíbles. Anti-CCP: anticuerpo antipéptido cíclico citrulinado. C3 y C4: Componentes C3 y C4 del complemento. Fuente: creación propia

- NTIA sin causa específica, excepto pielonefritis bacteriana.

- NTIA en paciente inmunosuprimido por cualquier causa, excepto que se trate de pielonefritis bacteriana.

En la biopsia renal de una NTIA por medicamentos, el intersticio aparece ensanchado por edema, con un infiltrado inflamatorio principalmente de tipo mononuclear, predominantemente linfocitos y células plasmáticas. Hay presencia de eosinófilos en la mayoría de los casos; también puede haber macrófagos, neutrófilos y células gigantes multinucleadas, estos últimos más frecuentes en la causa infecciosa ${ }^{(44)}$.

En algunos casos, hay formación de granulomas no caseificantes. El infiltrado es principalmente focal (aunque puede ser difuso) y más prominente en la unión corticomedular, a menudo rodeando túbulos individuales. Se puede observar acúmulos de eosinófilos, pero si hay microabscesos de neutrófilos, se debe pensar en una causa infecciosa ${ }^{(20)}$.

Los túbulos presentan lesiones muy variables que pueden ser focales o difusas. En ocasiones hay franca necrosis tubular y, en otras, solo se ve una atenuación o pérdida del borde en cepillo de las células tubulares, las cuales pueden verse más basofílicas, descamando en la luz del túbulo o formando cilindros celulares. También se puede observar cambios regenerativos como la mitosis, núcleos grandes con nucleolos prominentes y binucleación, además se puede hallar infiltración de linfocitos o neutrófilos en el epitelio tubular, conocido como tubulitis.
En ocasiones puede identificarse una ruptura y aspecto laminado de la membrana basal tubular (MBT). Es muy importante evaluar si hay presencia de infección bacteriana, hecho diciente cuando hay predominancia de polimorfonucleares neutrófilos y bacterias en el gram. En los pacientes inmunosuprimidos es fundamental realizar tinciones especiales en la búsqueda de infecciones oportunistas como los virus (citomegalovirus, adenovirus y virus BK), las micobacterias, hongos o parásitos.

En Ias causas autoinmunes, la inmunofluorescencia es muy importante, en la que puede haber fuerte tinción lineal difusa de la MBT para IggG, con tinción variable de C3. En los casos crónicos predomina la fibrosis en el intersticio, lo cual es un siono de un mal pronóstico para la recuperación de la función renal (NTI crónica) ${ }^{(20)}$.

\section{TRATAMIENTO DE LA NTIA}

Una vez el diagnóstico de NTIA es establecido, el tratamiento depende de la causa específica que causó el daño. En los casos de infecciones, el manejo antimicrobiano diriogido es fundamental. En los pacientes inmunosuprimidos por fármacos hay que disminuir la inmunosupresión. Cuando se trata de medicamentos o productos herbales, la suspensión temprana de la sustancia implicada es muy importante para mejorar los desenlaces. En las causas autoinmunes, la evaluación sistémica del paciente y el manejo inmunosupresor guiado es clave para el control de la enfermedad. 
En todos los casos la correcta reanimación hídrica del paciente es imprescindible ${ }^{(45)}$.

Dado el mecanismo inmunológico subyacente de la NTIA, la terapia esteroidea se ha venido implementando como una estrategia de primera línea para el manejo de ella con etiología medicamentosa, herbal o autoinmune ${ }^{(38)}$. De lo anterior, es esencial tener en cuenta que los glucocorticoides pueden modular las reacciones de hipersensibilidad que ocurren en el intersticial renal, controlando así la inflamación y disminuyendo la fibrosis.

Con el objetivo de evaluar la evidencia disponible sobre el uso de esteroides en pacientes con diagnóstico de la NTIA secundaria a medicamentos, se realizó una búsqueda de la literatura en las principales bases de datos para determinar si existen guías en este tema. No se encontró ningún estudio aleatorio controlado que evaluara el uso de esteroides en pacientes con la NTIA. Solo se encontró algunos estudios retrospectivos con resultados contradictorios (Tabla 3) ${ }^{(13,46-50)}$.
Clarkon et al., publicaron en el 2004 una serie de 60 casos de la NTIA confirmados por biopsia renal. Un total de 36 pacientes recibieron terapia esteroidea y no se observó una mejoría siognificativa en la función renal (creatinina sérica) en 1, 6 o 12 meses de seguimiento ${ }^{(46)}$. Por el contrario, González et aI., reportaron los resultados observados en 52 individuos con diagnóstico histopatológico de la NTIA que recibieron terapia esteroidea, mostrando una mejoría siognificativa de la función renal y suspensión de la terapia dialítica cuando la inmunosupresión esteroidea fue iniciada en las primeras dos semanas posteriores al evento ${ }^{(47)}$. Prendecki et al., describen una cohorte de 58 pacientes con diagnóstico de NTIA que recibieron esteroides, los cuales fueron emparejados con controles 1:1 (29 casos y 29 controles), en este estudio se observó un efecto benéfico en el grupo que recibió terapia esteroidea, con una mejoría de la función renal a los dos años del seguimiento, en comparación con los que no recibieron esta terapia ${ }^{(49)}$.

Tabla 3. Estudios en donde se ha evaluado el uso de esteroides en pacientes con la NTIA

\begin{tabular}{|c|c|c|c|c|c|c|}
\hline \multirow{2}{*}{ Series } & \multicolumn{2}{|c|}{ Total pacientes } & \multicolumn{2}{|c|}{ Última creatinina (mg/dl) } & \multicolumn{2}{|c|}{ No recuperación función renal } \\
\hline & Casos* & Control* & Casos* & Controles* & Casos* & Controles* \\
\hline Clarkson et al. 2004. (46) & 26 & 16 & $\begin{array}{c}1,63 \\
(1,2-2,4)\end{array}$ & $\begin{array}{c}1,60 \\
(1,3-2,1)\end{array}$ & No dato & No dato \\
\hline González et al. 2008 (47) & 52 & 9 & $\begin{array}{c}2,1 \\
(0,7-12,7)\end{array}$ & $\begin{array}{c}3,7 \\
(0,7-8,9)\end{array}$ & $2(3,8 \%)$ & $4(44,4 \%)$ \\
\hline Raza et al. 2012 (48) & 37 & 12 & $\begin{array}{c}2,8 \\
(0,9-7,3)\end{array}$ & $\begin{array}{c}3,4 \\
(0,9-8,6)\end{array}$ & & \\
\hline Muriithi et al. 2014 (13) & 83 & 12 & $\begin{array}{c}1,4 \\
(1,1-1,8)\end{array}$ & $\begin{array}{c}1,5 \\
(1,1-2,6)\end{array}$ & $8 / 66(12 \%)$ & $1 / 6(17 \%)$ \\
\hline Valluri et al. 2015 (50) & 73 & 51 & & & $10 \%$ & $10 \%$ \\
\hline Prendecki et al. 2017 (49) & 29 & 29 & GFR $40 \mathrm{ml} / \mathrm{min}$ & GFR $30 \mathrm{ml} / \mathrm{min}$ & $6,9 \% * *$ & $20,6 \% * *$ \\
\hline
\end{tabular}

Entre las explicaciones propuestas para estos hallazgos discordantes están la no aleatorización de los pacientes, la inclusión de aquellos con gran deterioro de la función renal al momento del diagnóstico, las causas diversas de la NTIA (no todas medicamentosas), no todos los casos documentados con biopsia, el inicio tardío de la terapia esteroidea y la heterogeneidad en las dosis de esteroides utilizadas, entre
Otras ${ }^{(46,50,51-54)}$. Sin embargo, según la recomendación de los expertos, los esteroides son una opción terapéutica benéfica cuando hay un diagnóstico certero de la NTIA por medicamentos (idealmente confirmada por biopsia) ${ }^{(1,17,55)}$, con deterioro de la función renal que no mejora con la suspensión del medicamento o sustancia implicada y, cuando se inician en un período menor a dos semanas (ideal antes de una 
semana) y antes de que ocurran los cambios fibróticos renales ${ }^{(1,56)}$.

\section{Terapia esteroidea recomendada}

La dosis y frecuencia de administración de los esteroides es desconocida. En nuestro Hospital se utiliza el protocolo del Hospital Universitario 12 de Octubre de Madrid con buenos resultados (Figura 1) ${ }^{(47)}$. Otros esquemas con glucocorticoides se han aplicado con resultados similares.

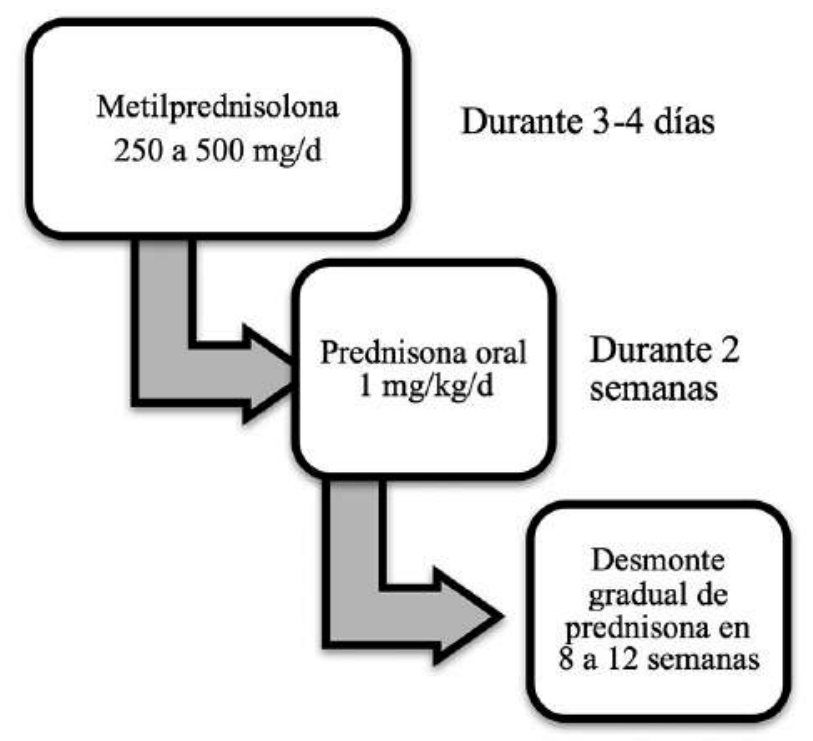

Figura 1. Esquema de la terapia con esteroides descrita por el Hospital Universitario 12 de Octubre de Madrid. Fuente: González et al

Posterior al inicio de la terapia esteroidea, la respuesta inicial se observa usualmente de manera temprana. Si a los 7 días de iniciado el manejo no hay mejoría, se debe suspender la terapia.

\section{Otras terapias}

Hay experiencia limitada con el uso de otras terapias inmunosupresoras en el manejo de la NTIA resistente o dependiente al manejo corticoideo. Algunos reportes de caso han utilizado micofenolato mofetil, ciclosporina e, incluso, ciclofosfamida con desenlaces variables (57,58). La utilización de micofenolato podría considerarse en pacientes con la NTIA confirmada por biopsia, dependientes de la terapia esteroidea o, con un tiempo de terapia mínimo a 6 meses posterior al diagnóstico ${ }^{(57)}$.

\section{PRONÓSTICO}

La recuperación de la función renal sin secuelas se observa en aproximadamente el $60 \%$ de los casos. Sin embargo, esto está determinado por el tipo de insulto que precipitó la lesión intersticial, la terapia recibida e incluso las condiciones idiosincráticas del paciente (22). La gran mayoría de los pacientes con la NTIA por meticilina presentan un buen pronóstico, a diferencia de otras etiologías y medicamentos en donde la recuperación es mucho menor ${ }^{(51,59)}$.

Entre el 5 al $10 \%$ de los pacientes con la NTIA se presenta un daño renal irreversible y del 1 al $10 \%$ de todos los casos requieren una terapia de reemplazo renal permanente ${ }^{(46,60)}$. Del 20 al $40 \%$ de los pacientes tienen una recuperación incompleta con la elevación persistente de la creatinina sérica ${ }^{(46,60)}$. Entre los factores clínicos que se han asociado con el mal pronóstico se encuentran:

- Lesión renal prolongada por más de 3 semanas.

- NTIA persistente por AINES.

- Hallazgos histológicos intersticiales con fibrosis o atrofia tubular avanzada (> $50 \%$ ), o la presencia de compromiso granulomatoso ${ }^{(51)}$.

\section{CONCLUSIÓN}

La NTIA es una causa importante $y$ frecuente de la LRA con manifestaciones clínicas muy variadas y con etiología multifactorial, donde los medicamentos o productos herbales juegan un papel causal determinante que, con frecuencia, pasa desapercibido. Una sospecha clínica temprana y un tratamiento oportuno pueden mejorar el pronóstico de los pacientes para que no queden secuelas graves como la ERC.

\section{CONFLICTOS DE INTERESES}

Ninguno por declarar. 


\section{REFERENCIAS BIBLIOGRÁFICAS}

1. Surendra M, Raju S, Chandragiri S, Uppin MS, Raju $N$. Steroid therapy in drug induced acute interstitial nephritis-Retrospective analysis of 83 cases. Saudi J Kidney Dis Transpl. 2019;30(1):157-65.

2. Ulinski T, Sellier-Leclerc AL, Tudorache E, Bensman A, Aoun B. Acute tubulointerstitial nephritis. Pediatr Nephrol. 2012;27(7):1051-7. DOI 10.1007/s00467-0111915-9.

3. Kizilbash Q. Successful management of acute interstitial nephritis in two cases of disseminated tubercuIosis. Tuberculosis (Edinb). 2016;101S:S135-S6. DOI 10.1016/j.tube.2016.09.014.

4. Johnson R, Feehally J, Floege J, Tonelli M. Comprehensive Clinical Nephrology. 6ed. EE. UU: Elsevier; 2019. 1360 p.

5. Skorecki K, Chertow G, Marsden P, Taal M, You A. Brenner y Rector. El riñón. 10 ed. España: Elsevier; 2018.

6. Praga M, Gonzalez E. Acute interstitial nephritis. Kidney Int. 2010;77(11):956-61. DOI 10.1038/ki.2010.89.

7. Cameron JS. Allergic interstitial nephritis: clinical features and pathogenesis. Q J Med. 1988;66(250):97-115.

8. Pettersson E, von Bonsdorff $M$, Tornroth T, Lindholm H. Nephritis among young Finnish men. Clin Nephrol. 1984;22(5):217-22.

9. Eddy AA. Drug-induced tubulointerstitial nephritis: hypersensitivity and necroinflammatory pathways. Pediatr Nephrol. 2019. DOI 10.1007/s00467-019-04207-9.

10. Haas M, Spargo BH, Wit EJ, Meehan SM. Etiologies and outcome of acute renal insufficiency in older adults: a renal biopsy study of 259 cases. Am J Kidney Dis. 2000;35(3):433-47. DOI 10.1016/S02726386(00) $70196-X$.

11. Farrington K, Levison DA, Greenwood RN, Cattell WR, Baker LR. Renal biopsy in patients with unexplained renal impairment and normal kidney size. Q J Med. 1989;70(263):221-33.

12. Praga M, Sevillano A, Aunon P, Gonzalez E. Changes in the aetiology, clinical presentation and management of acute interstitial nephritis, an increasingly common cause of acute kidney injury. Nephrol Dial Transplant. 2015;30(9):1472-9. DOI 10.1093/ndt/gfu326.

13. Muriithi AK, Leung N, Valeri AM, Cornell LD, Sethi $S$, Fidler ME, et al. Clinical characteristics, causes and outcomes of acute interstitial nephritis in the elderly. Kidney Int. 2015;87(2):458-64. DOI 10.1038/ ki.2014.294.

14. Davison AM, Jones $\mathrm{CH}$. Acute interstitial nephritis in the elderly: a report from the UK MRC Glomerulonephritis Register and a review of the literature. Nephrol Dial Transplant. 1998;13 Suppl 7:12-6. DOI 10.1093/ ndt/13.suppl_7.12.

15. Lamy Pp. Renal effects of nonsteroidal antiinflammatory drugs. Heightened risk to the elderly? J Am Geriatr Soc. 1986;34(5):361-7. DOI 10.1111/j.15325415.1986.tb04319.x.

16. Wilson GJ, Kark AL, Francis LP, Hoy W, Healy HG, Mallett AJ. The increasing rates of acute interstitial nephritis in Australia: a single centre case series. BMC Nephrol. 2017;18(1):329. DOI 10.1186/s12882-0170747-7.

17. Reddy S, Salant DJ. Treatment of acute interstitial nephritis. Ren Fail. 1998;20(6):829-38. DOI 10.3109/08860229809045180.

18. Schwarz A, Krause PH, Kunzendorf U, Keller F, Distler A. The outcome of acute interstitial nephritis: risk factors for the transition from acute to chronic interstitial nephritis. Clin Nephrol. 2000;54(3):179-90.

19. Baker RJ, Pusey CD. The changing profile of acute tubulointerstitial nephritis. Nephrol Dial Transplant. 2004;19(1):8-11. DOI 10.1093/ndt/gfog464.

20. Arias LF. Nefropatología [Internet]. [Cited 2018 July 12]. Disponible en: https://cutt.ly/JePPH6p

21. Fogo A, Kashgarian M. Diagnostic Atlas of Renal Pathology. 3 ed. Reino Unido: Elsevier; 2016.

22. Raghavan R, Eknoyan G. Acute interstitial nephritis - a reappraisal and update. Clin Nephrol. 2014;82(3):14962. DOI $10.5414 / \mathrm{CN} 10838$.

23. Lucas GNC, Leitao ACC, Alencar RL, Xavier RMF, Daher EF, Silva Junior GBD. Pathophysiological aspects of nephropathy caused by non-steroidal anti-inflammatory drugs. J Bras Nefrol. 2019;41(1):124-30. DOI 10.1590/2175-8239-jbn-2018-0107.

24. Perazella MA, Markowitz GS. Druog-induced acute interstitial nephritis. Nat Rev Nephrol. 2010;6(8):461-70. DOI 10.1038/nrneph.2010.71.

25. Nast CC. Medication-Induced Interstitial Nephritis in the 21st Century. Adv Chronic Kidney Dis. 2017;24(2):72-9. DOI 10.1053/j.ackd.2016.11.016. 
26. Rho M, Perazella MA. Nephrotoxicity associated with antiretroviral therapy in HIV-infected patients. Curr Drug Saf. 2007;2(2):147-54. DOI 10.2174/157488607780598269.

27. Simpson IJ, Marshall MR, Pilmore H, Manley P, WiIliams L, Thein $\mathrm{H}$, et al. Proton pump inhibitors and acute interstitial nephritis: report and analysis of 15 cases. Nephrology (Carlton, Vic). 2006;11(5):381-5. DOI 10.1111/j.1440-1797.2006.00651.x.

28. Sierra F, Suarez M, Rey M, Vela MF. Systematic review: Proton pump inhibitor-associated acute interstitial nephritis. Aliment Pharmacol Ther. 2007;26(4):545-53. DOI 10.1111/j.1365-2036.2007.03407.x.

29. Ambruzs JM, Walker PD, Larsen CP. The histopathologic spectrum of kidney biopsies in patients with inflammatory bowel disease. Clin J Am Soc Nephrol. 2014;9(2):265-70. DOI 10.2215/CJN.04660513.

30. Gisbert JP, Gonzalez-Lama Y, Mate J. 5-Aminosalicylates and renal function in inflammatory bowel disease: a systematic review. Inflamm Bowel Dis. 2007;13(5):629-38. DOI 10.1002/ibd.20099.

31. Vasanth P, Parmley M, Torrealba J, Hamdi T. Interstitial Nephritis in a Patient with Inflammatory Bowel Disease. Case Rep Nephrol. 2016. DOI 10.1155/2016/4260365.

32. Chaponda M, Pirmohamed M. Hypersensitivity reactions to HIV therapy. Br J Clin Pharmacol. 2011;71(5):659-71. DOI 10.1111/j.13652125.2010.03784.x.

33. Effa EE, Ekrikpo UE, Borkum M, Rayner BL, Heering P, Okpechi IG. Clinical profile and outcome of patients with biopsy-proven acute interstitial nephritis in Cape Town: a 10-year review. Clin Nephrol. 2017;88(8):97104. DOI 10.5414/CN109163.

34. Lanot A, Bouvier N, Chatelet $\nabla$, Dina J, Bechade C, Ficheux $M$, et al. [BK virus infections in kidney transplantation]. Nephrol Ther. 2016;12(2):76-85. DOI 10.1016/j.nephro.2015.11.003.

35. Maripuri S, Grande JP, Osborn TG, Fervenza FC, Matteson EL, Donadio JV, et al. Renal involvement in primary Sjogren's syndrome: a clinicopathologic study. Clin J Am Soc Nephrol. 2009;4(9):1423-31. DOI 10.2215/CJN.00980209.

36. Serna Higuita LM, Londono J, Velez-Echeverri C, Serrano AK, Ochoa-Garcia C, Rojas Rosas L, et al. Síndrome de nefritis túbulo-intersticial y uveítis en una paciente pediátrica. NefroPlus. 2015;7(1):72-5. DOI 10.3265/NefroPlus.pre2015.Feb.12529.

37. Krishnan N, Perazella MA. Drug-induced acute interstitial nephritis: pathology, pathogenesis, and treatment. Iran J Kidney Dis. 2015;9(1):3-13.

38. Moledina DG, Perazella MA. Drug-Induced Acute Interstitial Nephritis. Clin J Am Soc Nephrol. 2017;12(12):2046-9. DOI 10.2215/CJN.07630717.

39. Yao YH, Lin CC, Chung YM, Yang AH, Li SY, Lin CC, et al. Tubulointerstitial nephritis and uveitis syndrome (TINU) with Fanconi's syndrome. Clin Nephrol. 2011;75 Suppl 1:75-8. DOI 10.5414/CN106471.

40. Karras DJ. Severe low back pain secondary to acute interstitial nephritis following administration of ranitidine. Am J Emerg Med. 1994;12(1):67-8. DOI 10.1016/0735-6757(94)90203-8.

41. Strutz F, Grupp C. [Tubulointerstitial Nephritis (DrugInduced, Inflammatory)]. Dtsch Med Wochenschr. 2018;143(11):802-5. DOI 10.1055/a-0511-8458.

42. Rosen S, Stillman IE. Acute tubular necrosis is a syndrome of physiologic and pathologic dissociation. J Am Soc Nephrol. 2008;19(5):871-5. DOI 10.1681/ ASN.2007080913.

43. Chawla LS, Kellum JA. Acute kidney injury in 2011: Biomarkers are transforming our understanding of AKI. Nat Rev Nephrol. 2012;8(2):68-70. DOI 10.1038/ nrneph.2011.216.

44. Brodsky S, Nadasdy T. En: Charles J, Vivette D, D’Agati, Jean L. Olson, Silva FG. Heptinstall's Pathology of the Kidney. 7 Ed. Toronto: Wolters Kluwer; 2015. p. 1111-65.

45. Chowdry AM, Azad H, Mir I, Najar MS, Ashraf BM, Muzafar WM, et al. Drug-induced acute interstitial nephritis: Prospective randomized trial comparing oral steroids and high-dose intravenous pulse steroid therapy in guiding the treatment of this condition. Saudi J Kidney Dis TranspI. 2018;29(3):598-607. DOI 10.4103/1319-2442.235171.

46. Clarkson MR, Giblin L, O'Connell FP, O’Kelly P, Walshe JJ, Conlon P, et al. Acute interstitial nephritis: clinical features and response to corticosteroid therapy. Nephrol Dial Transplant. 2004;19(11):2778-83. DOI 10.1093/ndt/gfh485.

47. Gonzalez E, Gutierrez E, Galeano C, Chevia C, de Sequera P, Bernis $C$, et al. Early steroid treatment improves the recovery of renal function in patients with 
drug-induced acute interstitial nephritis. Kidney Int. 2008;73(8):940-6. DOI 10.1038/sj.ki.5002776.

48. Raza MN, Hadid M, Keen CE, Bingham C, Salmon AH. Acute tubulointerstitial nephritis, treatment with steroid and impact on renal outcomes. Nephrology (Carlton, Vic). 2012;17(8):748-53. DOI 10.1111/j.14401797.2012.01648.X.

49. Prendecki M, Tanna A, Salama AD, Tam FW, Cairns T, Taube $\mathrm{D}$, et al. Long-term outcome in biopsy-proven acute interstitial nephritis treated with steroids. Clin Kidney J. 2017;10(2):233-9. DOI 10.1093/ckj/sfw116.

50. Valluri A, Hetherington L, McQuarrie E, Fleming $S$, Kipgen D, Geddes CC, et al. Acute tubulointerstitial nephritis in Scotland. QJM. 2015;108(7):527-32. DOI 10.1093/qjmed/hcu236.

51. Rossert J. Drug-induced acute interstitial nephritis. Kidney Int. 2001;60(2):804-17. DOI 10.1046/j.15231755.2001.060002804.x.

52. Clive DM, Stoff JS. Renal syndromes associated with nonsteroidal antiinflammatory drugs. $N$ Engl J Med. 1984;310(9):563-72. DOI 10.1056/ NEJM198403013100905.

53. Fernández-Juárez G, Pérez JV, Caravaca-Fontan F, Quintana L, Shabaka A, Rodriguez E, et al. Duration of Treatment with Corticosteroids and Recovery of Kidney Function in Acute Interstitial Nephritis. Clin J Am Soc Nephrol. 2018;13(12):1851-8. DOI 10.2215/ CJN.01390118.

54. Moledina DG, Perazella MA. Treatment of DrugInduced Acute Tubulointerstitial Nephritis: The
Search for Better Evidence. Clin J Am Soc Nephrol. 2018;13(12):1785-7. DOI 10.2215/CJN.12001018.

55. Porile JL, Bakris GL, Garella S. Acute interstitial nephritis with glomerulopathy due to nonsteroidal anti-inflammatory agents: a review of its clinical spectrum and effects of steroid therapy. J Clin Pharmacol. 1990;30(5):468-75. DOI 10.1002/j.1552-4604.1990. tb03487.x.

56. Woodruff AE, Meaney CJ, Hansen EA, Prescott GM. Azithromycin-Induced, Biopsy-Proven Acute Interstitial Nephritis in an Adult Successfully Treated with Low-Dose Corticosteroids. Pharmacotherapy. 2015;35(11):e169-74. DOI 10.1002/phar. 1660.

57. Preddie DC, Markowitz GS, Radhakrishnan J, Nickolas TL, D'Agati VD, Schwimmer JA, et al. Mycophenolate mofetil for the treatment of interstitial nephritis. Clin J Am Soc Nephrol. 2006; 1(4):718-22. DOI 10.2215/ CJN.01711105.

58. Zuliani E, Zwahlen H, Gilliet F, Marone C. Vancomycin-induced hypersensitivity reaction with acute renal failure: resolution following cyclosporine treatment. Clin Nephrol. 2005;64(2):155-8. DOI 10.5414/CNP64155.

59. Galpin JE, Shinaberger JH, Stanley TM, Blumenkrantz MJ, Bayer AS, Friedman GS, et al. Acute interstitial nephritis due to methicillin. Am J Med. 1978;65(5):75665. DOI 10.1016/0002-9343(78)90793-3.

60. Laberke HG, Bohle A. Acute interstitial nephritis: correlations between clinical and morphological findings. Clin Nephrol. 1980;14(6):263-73. 